\title{
ORIGIN OF MAGNETIC FIELDS IN JETS AND RADIO GALAXIES
}

\author{
William K. ROSE \\ Astronomy Program \\ University of Maryland \\ College Park, MD 20854, U.S.A.
}

\begin{abstract}
Jets are known to be emitted from the centers of active galaxies and quasars. In two recent papers (Rose, 1987, 1989) calculations of magnetic fields generated in jets because electrons drift with respect to protons have been described. Electron drift velocities can result from interaction with the cosmic background radiation or with ambient gas. We describe how magnetic fields can be generated in jets and radio galaxies.
\end{abstract}

\section{Introduction}

Collimated radio jets are found in a wide variety of extragalactic sources (e.g. radio galaxies and quasars). It is generally accepted that they are ejected from accretion disks surrounding massive black holes at the centers of active galaxies and quasars.

In two publications (Rose et al., 1984, 1987) we have studied some physical effects associated with the interaction between highly collimated relativistic jets and ambient gas. The initial interaction of a chargeneutralized jet traversing a collisionless plasma is through the twostream instability. For relevant physical conditions a relativistic jet loses energy primarily as the result of collisionless excitation of plasma waves, which heat the gas, and inverse Compton radiation. Both of these latter physical processes cause deceleration of electrons (and positrons) with respect to protons. The resultant currents generate magnetic fields.

\section{Generation of Magnetic Fields in Jets and Radio Galaxies}

The equation of jet hydrostatic equilibrium is

$$
-\nabla P+\frac{J}{C} \times B+n e E=0
$$

In Rose (1987, Paper I) we showed that, if pressure gradients can be neglected in equation (1) and the electric field assumed equal to zero except at the jet surface, then the coupled equations describing magnetic field generation are 


$$
\begin{aligned}
& \frac{1}{r} \frac{d}{d r}\left(r B_{\theta}(r)\right)=\frac{4 \pi}{c} n(r) \text { e } \xi(r) \frac{B_{z}(r)}{\left[B_{z}(r)^{2}+B_{\theta}(r)^{2}\right]^{1 / 2}} \\
& -\frac{d B_{z}(r)}{d r}=\frac{4 \pi}{c} n(r) \text { e } \xi(r) \frac{B_{\theta}(r)}{\left[B_{z}(r)^{2}+B_{\theta}(r)^{2}\right]^{1 / 2}}
\end{aligned}
$$

with $\xi^{2}(r)=v_{z}^{2}(r)+v_{\theta}^{2}(r)$. For $\xi(r)$ assumed known (e.g. $\xi(r)=$ constant) equations (2) and (3) can be solved numerically if two boundary conditions are given. In Paper I we described solutions to equations (2) and (3) subject to the boundary conditions $B_{z}=0$ at the jet surface (i.e. $r=R$ ) and $v_{z}=\xi(0)$ at $r=0$. Since the magnetic field is along the $z$ direction at the center of the jet electron velocity $v_{z}(0)$ is unaffected by the magnetic field and therefore determined by external forces on the electrons.

In Rose (1989, Paper II) we compared the Bessel function and helical solutions to numerical solutions of equations (2)-(3) with $n(r) \xi(r)$ set equal to a constant and physical boundary conditions imposed. This comparison showed that numerical solutions were approximately helical solutions at the center of the jet and more nearly approximated by Bessel functions in the outer regions.

The relativistic motions of jets imply that Compton energy losses caused by the interaction of electrons and the $2.7 \mathrm{~K}$ cosmic background radiation decelerate electrons with respect to protons. Since the electron drift velocity varies as $\mathrm{T}^{4}$ with $\mathrm{T}$ equal to the temperature of the cosmic background radiation (Paper $\mathrm{I}$ ) and $\mathrm{T}$ varies as $1+\mathrm{z}$ with $\mathrm{z}$ equal to the redshift it follows that the predicted electron drift velocity varies as $(1+z)^{4}$. In Paper II we assumed that jets consisted of Maxwell-Boltzmann distributions of electrons and protons and were characterized by a bulk velocity $v_{b}$ (or equivalently Lorentz factor $y$ ), opening angle $\Delta \theta$ and luminosity $\left(\mathrm{L} \simeq \pi \mathrm{R}^{2} \mathrm{~nm}_{\mathrm{p}} \mathrm{v}_{\mathrm{b}}{ }^{3} / 2\right.$ for mildly relativistic jets). The inverse Compton interaction between the $2.7 \mathrm{~K}$ background radiation and electrons in a mildly relativistic jet $(v=0.2 c)$ is sufficient to generate magnetic fields of $\sim 10^{-5} \mathrm{G}$ which are inferred to exist in the lobes of radio galaxies. There is observational evidence for extragalactic jets with $y \sim 10$. In Paper II we also described specific models for relativistic $(y=10)$ and ultrarelativistic $\left(y=10^{3}\right)$ jets in which magnetic field generation is entirely a consequence of interaction between jet electrons (and positrons) and the $2.7 \mathrm{~K}$ cosmic background radiation. Magnetic fields of $\geq 10^{-5} \mathrm{G}$ can be generated in high $\gamma$-jets.

\section{References}

Rose, W.K. (1987) Astrophys. J. 313, 146 (Paper I).

Rose, W.K. (1989) Astrophys. J. 337, 91 (Paper II).

Rose, W.K., Beall, J.H., Guillory, J. and Kainer, S. (1987) Astrophys. J. 314, 95.

Rose, W.K., Guillory, J., Beall, J.H. and Kainer, S. (1984) Astrophys. J. $280,550$. 